\title{
Experimental Study of a Microchannel Bubble Injector for Microgravity Applications
}

\author{
S. Arias · X. Ruiz • J. Casademunt • \\ L. Ramírez-Piscina · R. González-Cinca
}

Received: 15 October 2007 / Accepted: 15 April 2008

(C) Springer Science + Business Media B.V. 2008

\begin{abstract}
We perform a quantitative characterization of a microbubble injector in conditions relevant to microgravity. The injector pregenerates a slug flow by using a capillary $\mathrm{T}$-junction, whose operation is robust to changes in gravity level. We address questions regarding the performance under different injection conditions. In particular we focus on the variation of both gas and liquid flow rates. The injection performance is characterized by measuring bubble injection frequency and bubble sizes. We obtain two distinct working regimes of the injector and identify the optimal performance as the crossover region between them.
\end{abstract}

\section{S. Arias $(\bowtie)$}

Escola Politècnica Superior de Castelldefels,

Universitat Politècnica de Catalunya, Barcelona, Spain

e-mail: Santiago.arias@upc.edu

S. Arias · L. Ramírez-Piscina · R. González-Cinca Space and Aeronautics Research Centre,

CRAE, Barcelona, Spain

X. Ruiz

Área de Física Aplicada,

Universitat Rovira i Virgili Tarragona, Tarragona, Spain

X. Ruiz · J. Casademunt · L. Ramírez-Piscina

Institut d'Estudis Espacials de Catalunya,

IEEC, Barcelona, Spain

J. Casademunt

Departament d'Estructura i Constituents de la Matèria, Facultat de Física, Universitat de Barcelona,

Barcelona, Spain

L. Ramírez-Piscina · R. González-Cinca

Departament de Física Aplicada,

Universitat Politècnica de Catalunya, Barcelona, Spain
Keywords Bubble $\cdot$ Microgravity $•$ Multiphase flow $~$ Gas injection

\section{Introduction}

The formation and management of multiphase flows is a key aspect in some technologies for space applications. For example, bubbles or drops of one phase can be dispersed into a different phase in order to favour either energetic or mass exchange. In this context, it may be necessary to maximize the contact area between both phases, which implies the generation of large numbers of small bubbles or drops, with sizes which ideally should be subject to control. In the case of bubble generation, buoyancy alone is usually enough on Earth to produce detachment of bubbles from the injector (Kulkarni and Joshi 2005). However in microgravity environments this is not the case, and different methods have been devised to produce bubble detachment, such as the employ of cross-flow and co-flow configurations (Pampering and Rath 1995; Bhunia et al. 1998; Forrester and Rielly 1998), the application of fields (Di Marco et al. 2003; Iacona et al. 2006), etc. Performance of these methods was not completely satisfactory for certain purposes, in the sense that bubble sizes were typically too large, with large size dispersion, and the methods were sensitive to the gravity level.

Based on previous analyses of the detachment process (Triplett et al. 1999a, b; Liu et al. 2005), a new microinjector for generating small bubbles was recently proposed and tested, which addressed these issues with significant improvement (Carrera et al. 2008). In this injector, small gas bubbles were formed by the action of a liquid cross-flow in a capillary $\mathrm{T}$-junction, thus 
generating a slug flow. This slug flow was injected into a cavity, which resulted in a jet of bubbles of small size (of the order of the employed capillaries), with very small size dispersion, and insensitive to gravity level (Carrera et al. 2008).

The key point of this method is that one does not inject only air but a slug flow, that is, a controlled mixture of gas a liquid. A periodic train of bubbles is thus already formed before the actual injection, and the inertia of the injected flow is used in turn to spread the bubbles to form a jet were bubble coalescence is minimized. Note that if walls are completely wet by the liquid, capillary forces have no action against detachment of the bubbles from the injection tube into a cavity, contrary to other injection configurations. Indeed, for this injector the relevant processes controlling bubble formation (both bubble size and generation frequency) are those generating the slug flow, at the T-junction. In the nominal regime of such injector, capillary forces dominate over buoyancy, if present (small Bond number), and over inertial forces (small Weber number). Bubble formation thus results from the competition between capillary forces and the drag due to the liquid cross-flow (note that the drag can be large even in the small flux limit when the forming bubble occupies the available cross-section of the capillary) (Carrera et al. 2008). By balancing both forces the resulting bubble diameter $\phi_{B}$ in this regime was shown to be:

$\phi_{B}=\phi\left(\frac{1}{\alpha}-\frac{1}{2 \alpha^{2}}\left(\frac{W e}{W e^{c}}\right)^{1 / 2}\right)+O(W e)$

where $\phi$ is the capillary diameters, $\alpha$ is a geometrical factor and $W e^{c}$ a critical Weber number, both to be determined in experiments (Carrera et al. 2008), and the nominal cross-flow Weber number $W e$ is given by

$W e=\frac{\rho_{l} u_{l}^{2} \phi}{\sigma}$

being $\rho_{l}$ the liquid density, $\sigma$ the surface tension, and $u_{l}$ the mean velocity of the cross-flow of the liquid. Therefore in the small flow rates the bubble size is expected to be basically determined by the liquid capillary diameter, with a weak linear dependence on the mean velocity of the cross-flow of the liquid $u_{l}$ through the square root of the Weber number We.

In this paper we will address further experimental characterization of the injector under normal gravity conditions. Nevertheless, results in the two environments, normal gravity and microgravity conditions, do not show significant differences (Carrera et al. 2008).
Namely we will study the generation frequency and size of bubbles, as functions of injection parameters. To this end, large variations of both gas and liquid flow rates will be used. The objective will be not only to test the theoretical predictions, but to characterize the injector performance beyond the applicability regime of Eq. 1 . As a result we identify a second working regime, for larger flows, and find the optimum operating regime of the injector.

\section{Experimental Setup}

\section{Test Section}

The scheme of the experimental setup is shown in Fig. 1. The injector tested in this experiment was made of methacrylate, and pregenerates the slug flow by means of a T-junction formed by two $1 \mathrm{~mm}$ diameter capillaries. One capillary was fed by the air supply system. The second one (corresponding to the liquid crossflow) was fed by the water supply system. One high speed video camera with a CMOS sensor operating at 2000 frames-per-second, with a resolution of $640 \times$ 512 pixels, was focused at the T-junction to record the detachment and formation of the bubbles. The injector was designed with outer square cross-section to avoid optical distortions due to curved surfaces. Rear lighting was provided by a light source with 280 ultrabright white LED, 7000 mcd each one, and homogenized by a $60^{\circ}$ holographic diffuser sheet. Residual air and water coming from the injector were accumulated in a residual tank.

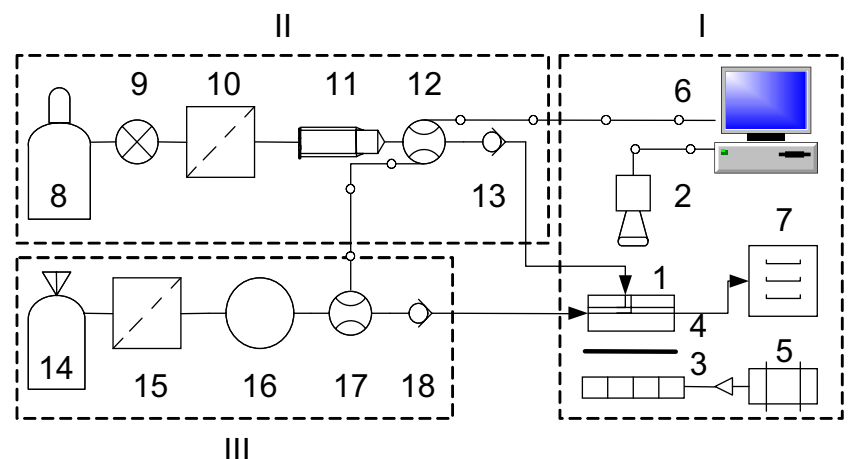

Fig. 1 Experimental equipment. $I$ test section (1 Injector, 2 HS camera, 3 light source, 4 diffuser, 5 power supply, 6 computer, 7 residual tank); $I I$ air supply system ( 8 air bottle, 9 manometer, 10 air filter, 11 chocked orifice, 12 air mass flow meter, 13 check valve); $I I I$ water supply system ( 14 water tank, 15 filter, 16 pump, 17 water mass flow meter, 18 check valve) 


\section{Air Supply System}

To generate bubbles synthetic and filtered air was used. Air was driven through the capillary under constant mass flow rate. The mass flow rate of air was controlled using a chocked orifice. The pressure of the air line before the chocked orifice pressure was regulated from 0 to 10 bars with an uncertainty less than 0.1 bars. The air flow rate was measured by a Bronkhorst Hi-Tec's air mass flow meter (F-201C9 series) under constant temperature, being $15^{\circ} \mathrm{C}$ and $1.225 \mathrm{~kg} / \mathrm{m}^{3}$ the values of temperature and density respectively. The uncertainties in the air flow rate measurements were less than $0.05 \mathrm{ml} / \mathrm{min}$.

\section{Water Supply System}

To inject the liquid cross-flow an Ismatec MCP-Z water pump was used. It was fed with distilled and filtered water from a water tank. The water flow rate was measured by a Bronkhorst Hi-Tec's Liquid-flow mass meter (L30 series) with uncertainties less than $0.05 \mathrm{ml} / \mathrm{min}$. The water volume range was changed from 11.83 to $36.50 \mathrm{ml} / \mathrm{min}$.

\section{Results and Discussion}

Experiments were performed at several liquid volumetric flow rates, under normal gravity conditions. For each value of $Q_{l}$ a large number of values of gas volumetric flow rates $Q_{g}$, ranging typically from 0.25

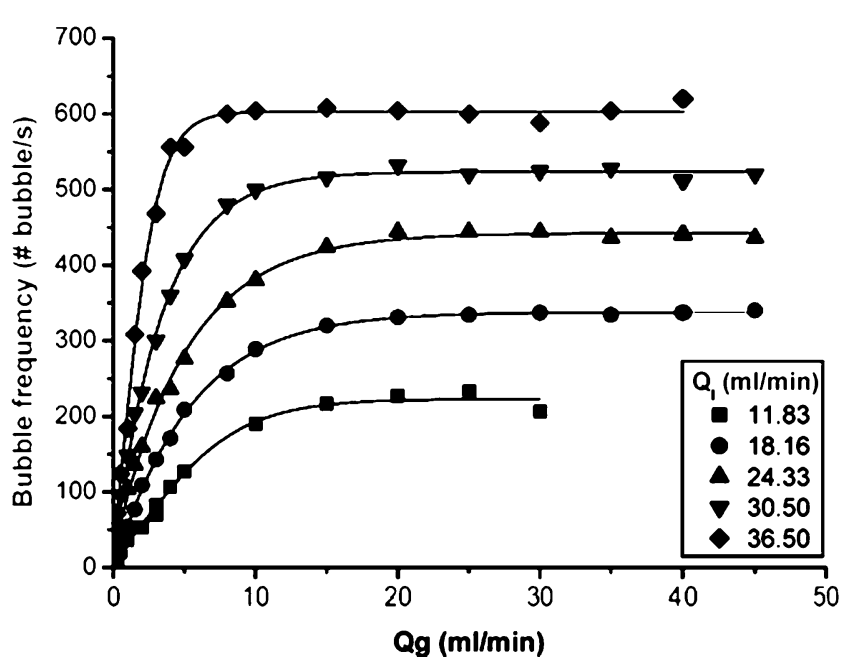

Fig. 2 Dependence of bubble frequency vs. air volumetric flow rate for different liquid volumetric flow rates; lines are guides to the eye. Symbols experimental results

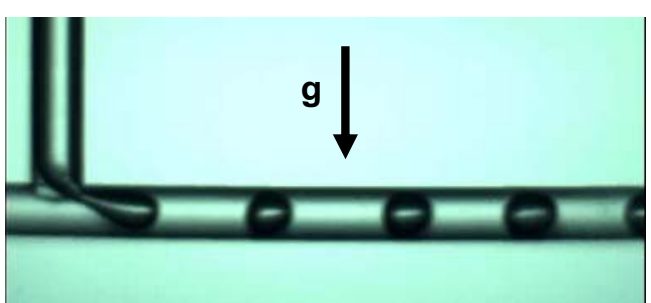

a)

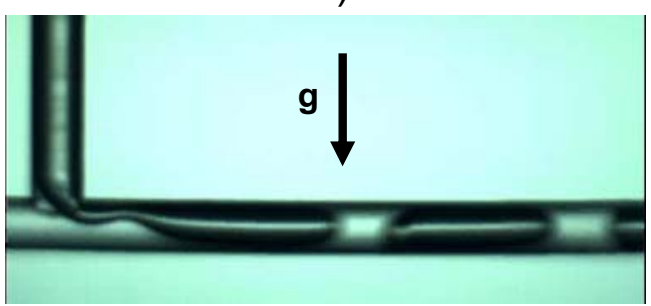

b)

Fig. 3 Slug flow at a low gas flow rate, $\mathbf{b}$ high gas flow rate

to $45 \mathrm{ml} / \mathrm{min}$, were employed. For each chosen couple of values $Q_{l}, Q_{g}$ images were taken by the high speed camera and recorded typically at $2000 \mathrm{fps}$. Analysis of the films permitted to measure the bubble generation frequency. This frequency was at most of the order 600 bubbles per second, and hence this process was well resolved by the image acquisition system. No frequency aliasing occurs in any case.

Results on bubble generation frequency are shown in Fig. 2. In this figure we see that, regardless of the liquid flow rate, two different regimes can be distinguished. In a first regime, corresponding to small gas fluxes, the generation frequency appears as a linear function of $Q_{g}$. For volumetric gas flow rates of the order of $10 \mathrm{ml} / \mathrm{min}$, a crossover to a saturation regime can be observed. At saturation, further increasing of gas flow rate does not imply larger generation frequencies, but larger bubbles instead (see Fig. 3). The asymptotic saturation values are used below in Fig. 4.

Regarding the homogeneity of bubble sizes, the low flow rate regime is characterized by a very small size dispersion. Also the timing of the bubble generation is very regular, in such a way that the generation frequency $f$ (number of generated bubbles per unit time) is very well defined. However, in the large flow rates regime, this regularity is lost. Both sizes and generation times present a larger dispersion that can be directly observed in the images.

We thus see that the slow flow rate regime presents the desired features of small and monodispersed bubble sizes. Therefore, the crossover to the saturation regime marks the optimal operation regime of the injector. In Fig. 4 we show the bubble generation frequency 


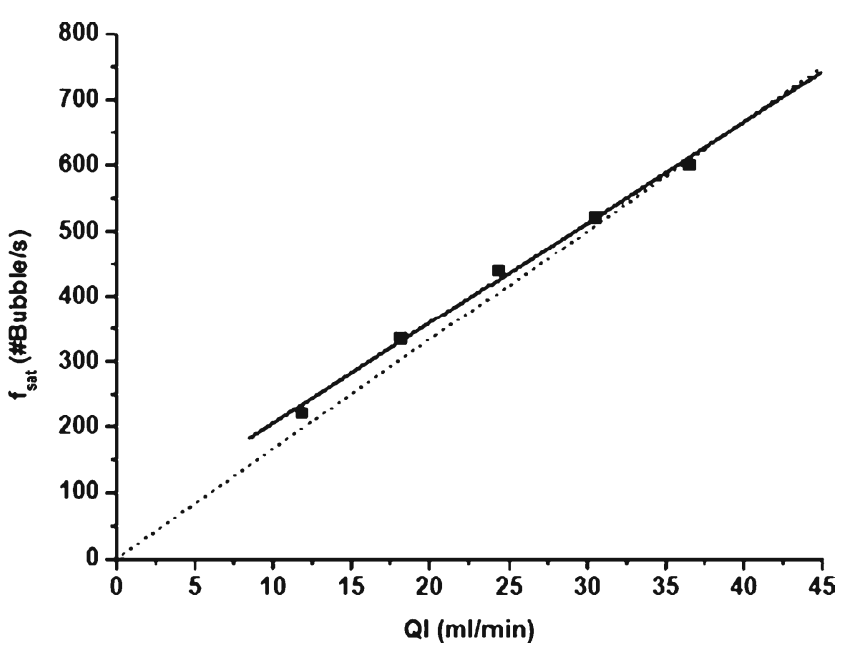

Fig. 4 Saturation frequency vs. liquid volumetric flow rate. Symbols experimental results, line linear fit, dot line theoretical prediction of $f_{\text {sat }}$

reached at saturation for each liquid flow rate. It is interesting to observe that a simple linear relation holds in its dependence on $Q_{l}$. The linear regression of the experimental results provides the fit: $f_{\text {sat }}=15.35 Q_{l}+$ 52.03 (in the figure units).

We can easily identify the first linear regime as the slow flow regime analysed in (Carrera et al. 2008). Images of the bubble formation process at the $\mathrm{T}$ (Fig. 3-a) show indeed that the bubble formation occur when its size is comparable to that of the capillary, regardless of how slow the fluxes are, in a way consistent with the theoretical considerations. Moreover Eq. 1 predicts a bubble size independent of $Q_{g}$ in this regime, which corresponds to a generation frequency proportional to $Q_{g}$ as seen in Fig. 2. The limit of this regime can be found by considering an important temporal scale in the bubble generation process, namely $Q_{l} / \phi^{3}$. This scale is proportional to $u_{l} / \phi$, the time needed by the liquid flow to cross a distance of the order of the capillary diameter, and hence should mark the minimum time necessary to form a bubble at the $\mathrm{T}$ junction. This scale gives then the saturation frequency for the generation process:

$$
f_{\text {sat }}=Q_{l} / \phi^{3}
$$

For the capillary used in our experiments $(\phi=1 \mathrm{~mm})$, saturation frequency should be proportional to $Q_{l}$, as observed in Fig. 4, with a proportionality constant of $1,000 \mathrm{~cm}^{-3}$. In the scales used in Fig. 4, we can then predict a slope of $16.67 \mathrm{~s}^{-1} /(\mathrm{ml} / \mathrm{min}$ ) (dot line in Fig. 4), which presents a good agreement with the results shown.

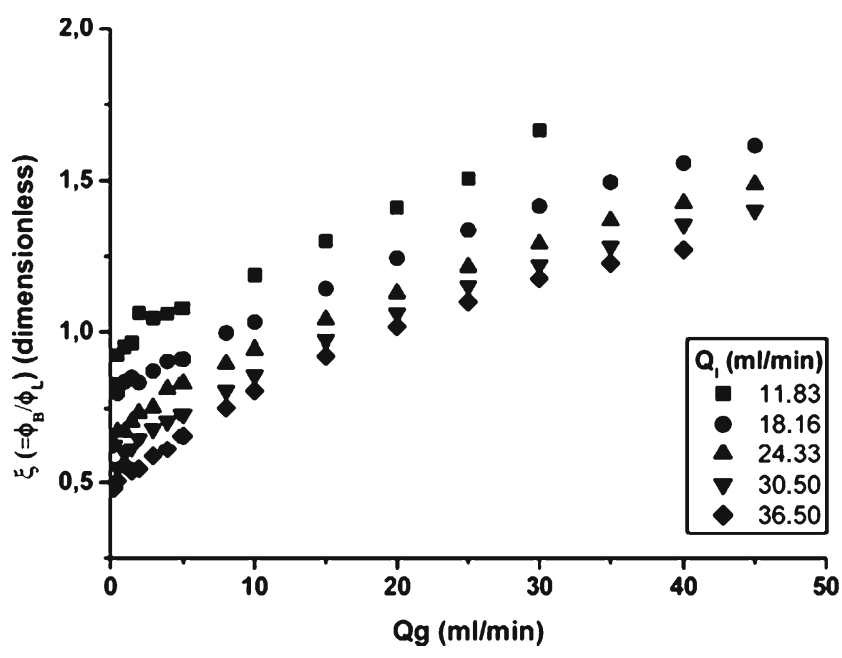

Fig. 5 Dependence of average bubble size vs. air flow rate

The mean size of the generated bubbles has been calculated by estimating the diameter of the equivalent sphere with the same volume as

$\phi_{B}=\left(\frac{6}{\pi} \frac{Q_{g}}{f}\right)^{1 / 3}$

We show this calculated bubble size in Fig. 5. Scaling this size with the liquid capillary diameter we obtain relative sizes of the order of 1 , thus demonstrating the excellent performance of the injector. In these results we see that bubble size is an increasing function of the gas flow rate. In fact one would expect a constant bubble size for small $Q_{g}$ (not clearly seen here due to experimental uncertainties, which are amplified in $\phi_{B}$ when both $Q_{g}$ and $f$ tend to zero), and a dependence on $Q_{g}^{1 / 3}$ in the saturation region. Regarding the effect

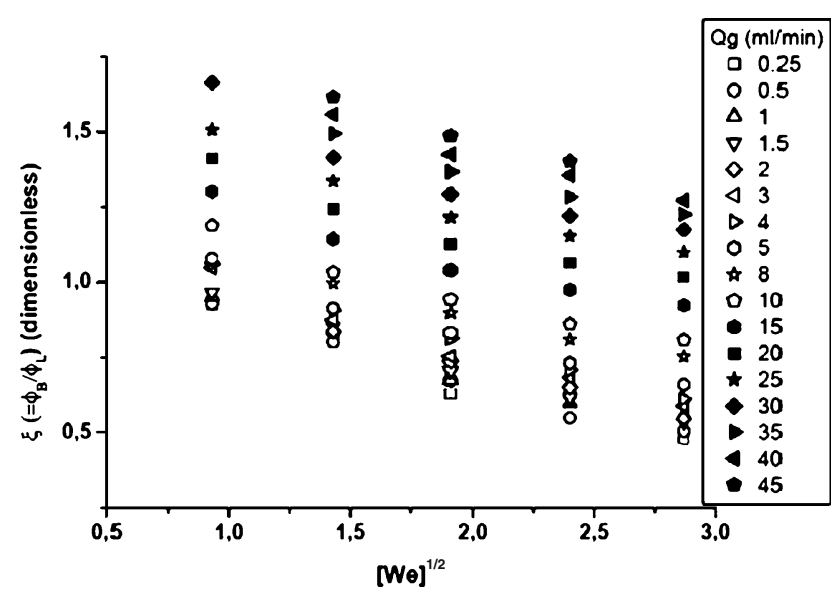

Fig. 6 Dimensionless bubble diameter vs. square root of Webber number 
of changes in liquid flow rates, we see that sizes are reduced when increasing $Q_{l}$. This is a common result in previous research of bubble generation using crossflow configurations.

Finally, we have checked the theoretical prediction available for the slow flow rate regime. To this end in Fig. 6 we have plotted in the scaled bubble size versus the square root of the nominal Weber number of the liquid cross-flow We. As we can see in the figure, bubble size depends linearly on $W e^{1 / 2}$. Points corresponding to the smaller gas flow rates (roughly up to $2 \mathrm{ml} / \mathrm{min}$ ), tend to superpose in the figure, consistently with the theoretical prediction that in this regime sizes are independent of $Q_{g}$ and depending on $W e^{1 / 2}$ only. From these points we can obtain a fit of the unknown parameters of the theory, $\alpha=0.86 \pm 0.06$ and $W e^{c}=$ $8.64 \pm 2.2$. Nevertheless, calculation of bubble sizes for small $Q_{g}$ are again subject to larger uncertainties, and the superposition of the points is not perfect.

\section{Conclusions}

We have performed an extensive characterization of the bubble injector proposed in Carrera et al. (2008), which is based on the generation of a slug flow in a T-junction prior to injection. This injector is thus analogous to other cross-flow configuration, but operates in the capillary regime (small Bond number) and hence its performance is independent of the gravity level. For this characterization we have taken images of the T-junction of the injector, where the slug flow is formed, and have measured the frequency of bubble formation for a broad range of injection rates of both gas and liquid. This frequency also permitted us to calculate mean bubble sizes.

Results indicate the presence of two distinct operation regimes of the injector. In a first regime, corresponding to small gas fluxes, generation frequency is linear with volumetric gas flow rate. For larger fluxes, frequency saturates whereas bubble size increases. For applications in which it is important to generate large number of small bubbles (to maximize the contact area between both phases) the limit of optimum operation will correspond to the region of crossover to saturation. The maximum frequency achieved for each liquid flux rate (saturation frequency) happens to depend linearly on $Q_{l}$, as can be predicted by scaling arguments.

Regarding the dependence of bubble diameter on the liquid flow rate, results show a linear behaviour on the square root of the nominal Weber number of the liquid cross-flow, as predicted by the theory. Furthermore, results are consistent with the prediction of independence of bubble size on gas flux rate for small $Q_{g}$.

As a final comment, we would like to remark that, by operating in the capillary regime, experiments on Earth can provide valuable information on the performance of this kind of injector relevant to its operation in microgravity. More experiments are still required to assess further issues concerning dispersion of bubble size and frequency, and changes of parameters, most remarkably the capillary diameters and of fluid parameters.

Acknowledgements We acknowledge financial support by Ministerio de Educación y Ciencia (Project numbers ESP200628459-E, FIS2006-03525 and FIS2006-11452-C03-02, Spain).

\section{References}

Bhunia, A., Pais, S.C., Kamotani, Y., Kim, I.: Bubble formation in a coflow configuration in normal and reduced gravity. AIChE J. 44, 1499-1509 (1998)

Carrera, J., Ruiz, X., Ramírez-Piscina, L., Casademunt, J., Dreyer, M.: Generation of a monodisperse microbubble jet in microgravity. AIAA Journal (2008, in press)

Di Marco, P., Grassi, W., Memoli, G., Takamasa, T., Tomiyama, A., Hosokawa, S.: Influence of electric field on single gasbubble growth and detachment in microgravity. Int. J. Multiph. Flows 29, 559-578 (2003)

Forrester, S.E., Rielly, C.D.: Bubble formation from cylindrical, flat and concave sections exposed to a strong liquid crossflow. Chem. Eng. Sci. 53, 1517-1527 (1998)

Iacona, E., Herman, C., Chang, S., Liu, Z.: Electric field effect on bubble detachment in reduced gravity environment. Exp. Therm. Fluid Sci. 31, 121-126 (2006)

Kulkarni, A.A., Joshi, J.B.: Bubble formation and bubble rise in gas-liquid system: a review. Ind. Eng. Chem. Res. 44, 5873$5931(2005)$

Liu, H., Vandu, C.O., Krishna, R.: Hydrodynamics of Taylor flow in vertical capillaries: flow regime, bubble rise velocity, liquid slug length and pressure drop. Ind. Eng. Chem. Res. 44, 4884-4897 (2005)

Pampering, O., Rath, H.J.: Influence of buoyancy on bubble formation at submerged orifices. Chem. Eng. Sci. 50, 3009-3024 (1995)

Triplett, K.A., Ghiaasiaan, S.M., Abdel-Khalik, S.I., Sadowdki, S.A.: Gas-liquid two-phase flow in microchannels. Part I: two-phase flow patterns. Int. J. Multiph. Flows 25, 377-394 (1999a)

Triplett, K.A., Ghiaasiaan, S.M., Abdel-Khalik, S.I., LeMouel, A., McCord, B.N.: Gas-liquid two-phase flow in microchannels. Part II: void fraction and pressure drop. Int. J. Multiph. Flows 25, 395-410 (1999b) 